\title{
Prolactin-stimulated survivin induction is required for beta cell mass expansion during pregnancy in mice
}

\author{
Yili Xu ${ }^{1}$ - Xiaojing Wang ${ }^{1} \cdot \mathrm{Li} \mathrm{Gao}^{1} \cdot \mathrm{Jiayu}^{\mathrm{Zhu}}{ }^{1} \cdot \mathrm{Hui}^{\mathrm{Zhang}}{ }^{1} \cdot \mathrm{Houxia} \mathrm{Shi}^{1}$ • \\ Minna $\mathrm{Woo}^{2} \cdot$ Xiaohong $\mathrm{Wu}^{1}$
}

Received: 22 February 2015 / Accepted: 26 May 2015 / Published online: 23 June 2015

(C) Springer-Verlag Berlin Heidelberg 2015

\begin{abstract}
Aims/hypothesis Prolactin(PRL)-stimulated beta cell proliferation is critical for maternal pancreatic beta cell mass expansion during pregnancy. However, the molecular effectors of the multiple putative signalling pathways downstream of the PRL receptor (PRL-R) are still elusive. Survivin has been shown to be induced during pregnancy. The aim of the present study was to define the essential role of survivin in gestational beta cell mass expansion.

Methods Expression of survivin was assessed in mouse islets during pregnancy and in insulinoma cells (INS-1) stimulated with PRL. Pregnant mice with targeted deletion of the survivin gene (also known as Birc5) in beta cells were assessed to determine the essential function of survivin in maternal beta cell mass expansion. INS-1 cells stimulated with PRL were used to explore the role of survivin in signalling pathways downstream of the PRL-R.

Results Survivin was significantly upregulated in maternal islets during pregnancy. With PRL stimulation, induction of survivin expression occurred predominantly in the nucleus
\end{abstract}

Yili Xu, Xiaojing Wang and Li Gao contributed equally to this article.

Electronic supplementary material The online version of this article (doi:10.1007/s00125-015-3670-0) contains peer-reviewed but unedited supplementary material, which is available to authorised users.

Xiaohong $\mathrm{Wu}$

drxhwu@njmu.edu.cn

1 Department of Endocrinology, First Affiliated Hospital with Nanjing Medical University, 300 Guangzhou Road,

210029 Nanjing, People's Republic of China

2 Toronto General Research Institute and Division of Endocrinology, Department of Medicine, University Health Network, University of Toronto, Toronto, ON, Canada and was associated with cell cycle progression to $\mathrm{S}$ and G2/M phase. Beta cell-specific survivin-knockout pregnant mice displayed glucose intolerance, attenuated beta cell mass expansion and impaired beta cell proliferation, with significant attenuation in the increased expression of $\mathrm{Cdk} 4 / \mathrm{Ccnd1}$, E2f1, p53 (also known as Trp53) and p21 (also known as $C d k n 1 a$ ) compared with wild-type controls during pregnancy. Targeted deletion of survivin in INS-1 cells resulted in cell cycle disturbance with an arrest in G1/S phase after PRL stimulation. Inhibitors of Akt, signal transducer and activator of transcription 5 (STAT5), PIM or extracellular signal-regulated kinase (ERK), significantly decreased the expression of survivin in PRL-stimulated INS-1 cells.

Conclusions/interpretation Survivin directly participates in PRL-mediated beta cell proliferation via Akt, STAT5-PIM and ERK signalling pathways during pregnancy.

Keywords Beta cell mass $\cdot$ Pregnancy $\cdot$ Prolactin $\cdot$ Signalling pathway $\cdot$ Survivin

$\begin{array}{ll}\text { Abbreviations } \\ \text { CCN } & \text { Cyclin } \\ \text { CDK } & \text { Cyclin-dependent kinase } \\ \text { ERK1/2 } & \text { Extracellular signal-regulated kinase 1 and } 2 \\ \text { FOXM1 } & \text { Forkhead box protein M1 } \\ \text { GSIS } & \text { Glucose-stimulated insulin secretion } \\ \text { HDAC1 } & \text { Histone deacetylase 1 } \\ \text { hGH } & \text { Human growth hormone } \\ \text { ITT } & \text { Insulin tolerance test } \\ \text { JAK2 } & \text { Janus kinase 2 } \\ \text { KO } & \text { Knockout } \\ \text { MAPK } & \text { Mitogen-activated protein kinase } \\ \text { NP } & \text { Non-pregnant } \\ \text { PDL } & \text { Pancreatic duct ligation }\end{array}$


PI3K Phosphatidylinositol 3-kinase

PRL Prolactin

PRL-R PRL receptor

siRNA Small interfering RNA

STAT5 Signal transducer and activator of transcription 5

WT Wild-type

\section{Introduction}

To meet increased physiological demands during pregnancy, maternal pancreatic beta cell mass expands significantly in both rodents and humans with enhanced insulin secretion per beta cell and increased beta cell proliferation and/or neogenesis [1-4]. Defects in compensatory beta cell mass expansion may therefore result in gestational diabetes. Clinical evidence shows that $70 \%$ of women with gestational diabetes develop type 2 diabetes later in life [5]. Exploring the adaptive capability of beta cells to increased metabolic load is therefore an important area of research aimed at preventing both gestational and type 2 diabetes. During pregnancy, the peak in beta cell proliferation coincides with increased prolactin (PRL) and/or placental lactogen level, suggesting that these lactogenic hormones may be vital for enhanced beta cell proliferation and function during pregnancy [2]. PRL (and the PRL receptor [PRL-R]) exerts its biological effects mainly by activating the Janus kinase 2 (JAK2)-signal transducer and activator of transcription 5 (STAT5), IRS1/2, phosphatidylinositol 3-kinase (PI3K) and mitogen-activated protein kinase (MAPK) pathways, leading to increased beta cell proliferation, survival and size [6-10]. The molecular effectors of the multiple putative signalling pathways downstream of the PRL-R are still elusive.

Survivin (also known as baculoviral inhibitor of apoptosis repeat containing protein 5 [BIRC5]) is a multifunctional protein that intersects the fundamental network of cellular processes, including cell death, cell division and cellular adaptation [11-13]. Our previous study revealed that transient perinatal expression of survivin is essential for pancreatic beta cell remodelling. Specific deletion of the survivin gene (Birc5) in beta cells impairs beta cell mass expansion, resulting in diabetes later in adulthood $[14,15]$. Female mice with survivin deletion (RIP-Cre-Survivin ${ }^{\mathrm{fl} / \mathrm{fl}}$ mice) showed a defect in beta cell mass expansion with insufficient insulin secretion and glucose intolerance after partial pancreatic duct ligation (PDL) [16]. Taken together, these findings suggest that survivin appears to exhibit a preferential requirement for proliferation of pre-existing beta cells during normal beta cell growth and at times of increased metabolic demand.

Recently, the mRNA level of survivin has been found to be elevated in gestational islets and PRL-stimulated cultured islets $[17,18]$. In the current study, we further explored the pattern of survivin expression in mouse maternal islets during pregnancy, finding that survivin was induced with increased nuclear localisation in response to PRL and this was associated with $\mathrm{S}$ and G2/M cell cycle progression via Akt, STAT5PIM and extracellular signal-regulated kinase (ERK) signalling pathways. Pregnant RIP-Cre-Survivin ${ }^{\mathrm{fl} / \mathrm{fl}}$ mice displayed glucose intolerance with impaired beta cell proliferation and mass expansion when compared with wild-type controls. Our data demonstrate that PRL-stimulated survivin induction plays a critical role in beta cell adaptation during pregnancy.

\section{Methods}

Mice RIP-Cre-Survivin ${ }^{\mathrm{fl} / \mathrm{fl}}$ mice were provided by H. Okada, Ontario Cancer Institute, Toronto, ON, Canada. Genotyping of RIP-Cre-Survivin ${ }^{\mathrm{fl} / \mathrm{fl}}$ (knockout, $\mathrm{KO}$ ) and RIP-Cre-Survivin ${ }^{+/+}$ (wild-type, WT) mice has been described previously $[14,16]$. Both C57BL/6 mice and littermate WT mice were used as controls. Day 0.5 of pregnancy was defined as the day on which vaginal plugs were found. All pregnant female mice were randomly divided into five groups: pregnancy day 10.5 (P10.5), P14.5 and P18.5 and after parturition day 4 (AP4) and AP8. Age-matched non-pregnant (NP) female mice were used as NP controls. Glucose tolerance tests, insulin tolerance tests (ITTs) and measurement of glucose-stimulated insulin secretion (GSIS) were performed as previously described [19]. Animal care and experimental protocols were approved by the Nanjing Medical University Animal Care Committee.

Islet isolation Pancreatic islets were isolated from control and KO female mice during pregnancy as described previously [20]. The GSIS of islets from NP and P14.5 mice was measured as previously described [21]. For the PRL stimulation study, islets were grown in an immunofluorescence confocal dish (Corning, Corning, NY, USA) and treated with PRL (Sigma, St Louis, MO, USA) (50 ng/ml) in culture medium for $24 \mathrm{~h}$. Double immunolabelling for insulin and Ki67 (Cell Signaling Technology, Beverly, MA, USA) was assessed.

Cell culture Insulinoma cells (INS-1) were provided by X. Han, Nanjing Medical University, Nanjing, China, and were cultured in RPMI-1640 medium with L-glutamine containing 10\% (vol./vol.) FBS. After cells reached near-confluence, the following experiments were undertaken.

For the PRL stimulation study, INS-1 cells were deprived of serum for $12 \mathrm{~h}$ and treated with PRL $(50 \mathrm{ng} / \mathrm{ml}, 500 \mathrm{ng} / \mathrm{ml})$ in culture medium for $24 \mathrm{~h}$. Cell protein lysates were collected for western blot analysis at indicated times. To inhibit the PRL signalling pathways, cells were treated with MK-2206 $2 \mathrm{HCl}$ (1 $\mu \mathrm{mol} / 1,48$ h), SH-4-54 (25 $\mu \mathrm{mol} / 1,72 \mathrm{~h})$, SGI-1776 (1 $\mu \mathrm{mol} / 1,48 \mathrm{~h})$ (Selleckchem, Houston, TX, USA) or U0126 (10 $\mu \mathrm{mol} / 1,30 \mathrm{~min})$ (Sigma, Oakville, ON, Canada) prior to PRL $(50 \mathrm{ng} / \mathrm{ml})$ treatment. Proteins were then harvested for western blot analysis. 
For the survivin small interfering RNA (siRNA) transfection study, INS-1 cells were transfected with the survivin gene, scrambled negative control or silencer select siRNA (sense 5'CCGAGAAUGAGCC UGAUUUdTdT-3' and antisense 5'dTdTGGCUCUUACUCGGACUAAA-3'[RiboBio, Guangzhou, China]) by Lipofectamine 2000 (Invitrogen of Thermo Fisher Scientific, Waltham, MA, USA) according to manufacturer's transfection protocol.

Flow cytometry Freshly isolated islets were dispersed with $0.05 \%$ (wt/vol.) trypsin-EDTA (Gibco of Thermo Fisher Scientific, Waltham, MA, USA). Cell suspensions $\left(1 \times 10^{6}\right.$ cells/islets sample or $2 \times 10^{6}$ cells/INS- 1 sample) were fixed in $80 \%$ (vol./vol.) ethanol. Pellets were resuspended in PBS containing propidium iodide and RNaseA (BD Pharmingen, San Diego, CA, USA). Cell cycle analyses were performed with a flow cytometer and FACSDiva software (Becton Dickinson, New Jersey, USA).

Quantitative real-time PCR Total RNA was isolated from islets and INS-1 cells using TRIzol reagent (Invitrogen). The cDNA was synthesised from $1 \mu \mathrm{g}$ RNA using PrimeScript RT Master Mix (TaKaRa, Otsu, Shiga, Japan). Primers used are listed in electronic supplementary material (ESM) Tables 1 and 2. Quantitative real-time PCR was performed as previously described [16].

Western blotting Islets and INS-1 cells were lysed and the protein concentration of the supernatant fractions was assayed by the BCA method using the BCA assay (Bio Rad, Berkeley, CA, USA). Extraction of nuclear and cytoplasmic proteins from INS-1 cells was carried out as previously described [22]. Protein, $30 \mu \mathrm{g}$ per lane, was separated on a $12 \%$ SDSPAGE gel. Gels were transferred to polyvinylidene difluoride membranes and then blotted with antibodies against survivin (Novus Biologicals, Littleton, CO, USA), Forkhead box protein M1 (FOXM1) (Santa Cruz Biotechnology, Santa Cruz, CA, USA), Akt, phosphorylated Akt (Ser473)(p-Akt), STAT-5, PIM-1, ERK1/2, phosphorylated ERK1/2 (p-ERK1/ 2) and GAPDH (Cell Signaling Technology), phosphorylated STAT-5 (p-STAT5) (Abcam, Cambridge, MA, USA) and histone deacetylase 1 (HDAC1) (Thermo Fisher, Waltham, MA, USA). Specific protein bands were visualised using commercial enhanced chemiluminescence reagents, with exposure by ChemiDocXRS system (Bio Rad). Densitometric quantification of protein bands was analysed using Image $J$ software version 1.37 (http://imagej.nih.gov/ij/). Samples were normalised to GADPH. At least five mice per group were analysed.

Immunofluorescence and morphometric analyses Insulin and glucagon immunostaining were performed as described previously [14]. According to the nucleus numbers, islets were divided into small islets (1-10 nuclei) and larger islets (>10 nuclei). Beta cell proliferation was assessed by double immunolabelling for insulin and Ki67 (Cell Signaling Technology). Beta cell apoptosis was detected by insulin and TUNEL co-staining using the In Situ Cell Death Detection Kit (Roche, Indianapolis, IN, USA). Morphometric analysis was performed using a fluorescent microscope system (Leica, Wetzlar, Germany).

Statistical analysis Data are presented as means \pm SEM and were analysed by unpaired Student's $t$ test and one-way analysis of variance with the post hoc Tukey test. Differences were considered to be statistically significant when $p<0.05$.

\section{Results}

Upregulation of survivin expression with pregnancyassociated increase in islet cell mass During pregnancy, increased maternal pancreatic beta cell mass and insulin secretion are required for elevated metabolic demands [23, 24]. Our data showed that beta and alpha cell mass progressively increased during pregnancy in $\mathrm{C} 57 \mathrm{BL} / 6$ mice, both peaking at P18.5, increasing about 2.1-fold and 1.6-fold, respectively, and returned back to NP levels at AP8 (Fig. 1a). Significant increment was observed in both islet cell number and islet size (Fig. 1b, c). At P18.5, beta and alpha cell number increased 1.7-fold and 1.2-fold, respectively, compared with the number of cells observed in the NP state, and similar increment in cell size was observed. Cell cycle analysis of isolated islets revealed that cell proliferation peaked at P14.5 (Fig. 1d-f). At P14.5 there was a markedly decreased number of cells in the G0/G1 phase and significantly increased number of cells in the $\mathrm{G} 2 / \mathrm{M}$ phase, returning back to the NP state after parturition. The mRNA levels of survivin progressively increased during pregnancy, peaking at P14.5, and returned back to the NP level after parturition (Fig. 1g). There was no detectable survivin protein in the NP state but survivin became evident at P10.5; levels peaked at P14.5, declined by P18.5 and became (and remained) undetectable by AP4 and AP8 (Fig. 1h).

PRL-stimulated survivin expression in INS-1 cells Pregnancy-associated beta cell mass expansion is mainly stimulated by the elevation of circulating pregnancy hormones, including growth hormone, PRL and placental lactogen $[25,26]$, among which PRL plays a key role [26, 27]. To determine whether the rise in survivin levels with increased beta cell proliferation was dependent on PRL, we examined survivin expression in INS-1 cells in response to different concentrations of PRL. The percentage of cell number in G0/G1 phase significantly decreased after PRL stimulation in a concentration-dependent manner (Fig. 2a), while that in S and G2/M phases increased markedly (Fig. 2b, c). 
Fig. 1 Increased islet cell mass and survivin expression during pregnancy in mice. (a-f) Beta and alpha cell mass (a), cell number (b), cell size (c) and cell cycle analyses of islet cells (d-f) from mice in the NP state and at P10.5, P14.5, P18.5, AP4 and AP8. White bars, beta cells; black bars, alpha cells. (g, h) Quantitative real-time PCR analysis of survivin (g) and western blot analysis of survivin protein (h) in isolated islets at above time points. The data are presented as means $\pm \operatorname{SEM}(n=5) . * p<0.05$ and ${ }^{* *} p<0.01$ vs NP; ${ }^{\dagger} p<0.05$ and ${ }^{\dagger \dagger} P<0.01$ vs $P 10.5$
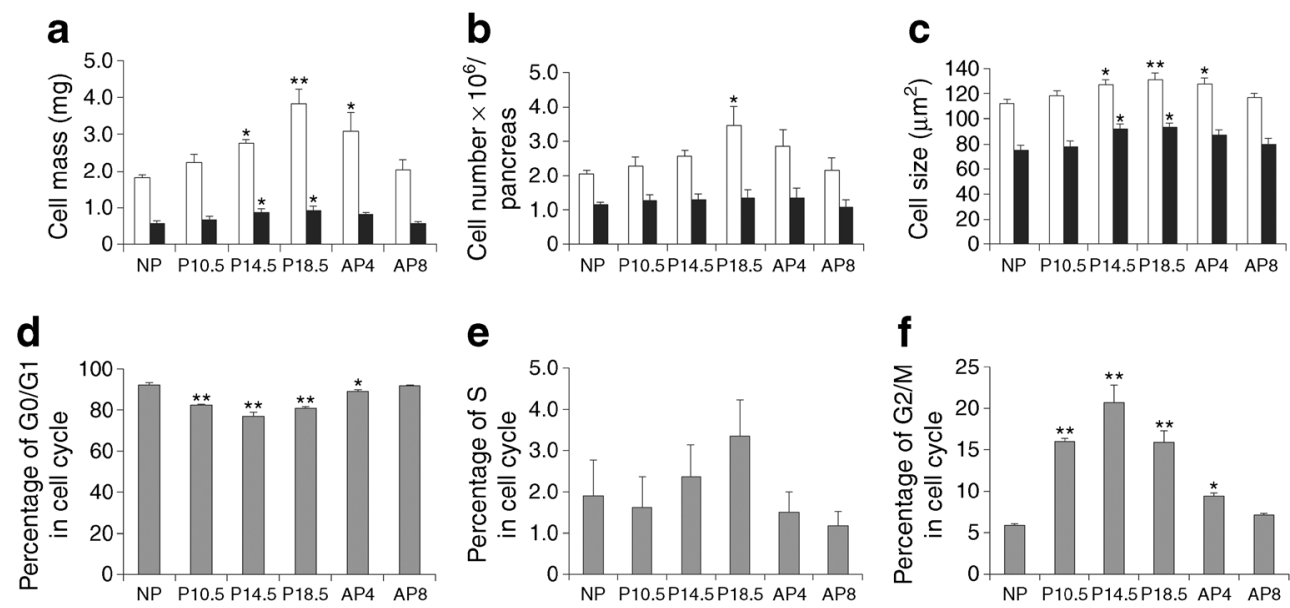
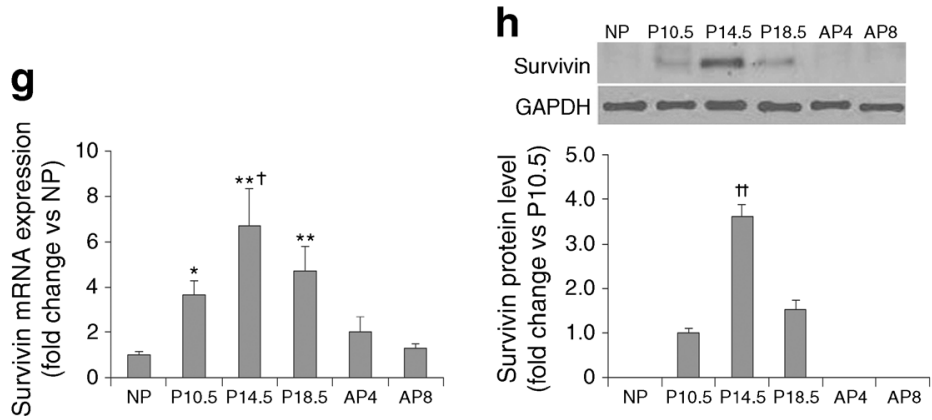

Along with these changes, the levels of survivin protein increased significantly upon PRL stimulation (Fig. 2d). As survivin is expressed in different subcellular compartments to mediate diverse cellular processes [12], we further examined the effect of PRL on the localisation of survivin in different subcellular compartments. In response to PRL stimulation, an increase in survivin was observed in both the nuclear and the cytoplasmic compartment, with the more marked increase occurring in the nucleus as determined by cell fractionation quantification (Fig. 2e). These data indicate that the induction in nuclear survivin expression could be regulated by PRL, resulting in INS-1 cell proliferation.
Fig. 2 Survivin expression in INS-1 cells after PRL stimulation. (a-d) Cell cycle analyses (a-c) and western blot analysis of survivin (d) in INS-1 cells after stimulation with PRL for $24 \mathrm{~h}$. (e) Western blots and quantification showing the nuclear $(\mathrm{N})$ and cytoplasmic (C) level of survivin protein in INS-1 cells with (black bars) and without (control, white bars) PRL (50 ng/ml, black bars) stimulation for $24 \mathrm{~h}$. GAPDH and HDAC1 were used as controls.

The data are presented as means \pm SEM $(n=8) .{ }^{*} p<0.05$ and $* * p<0.01$ vs control
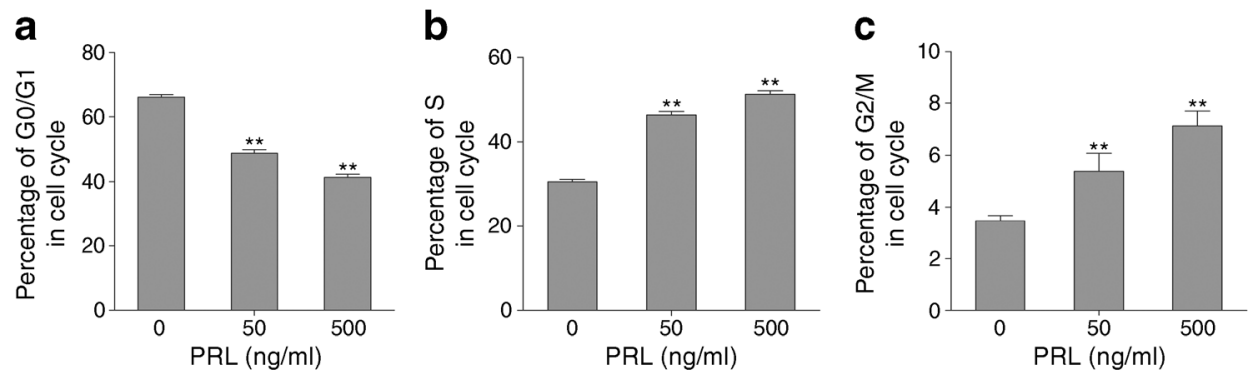
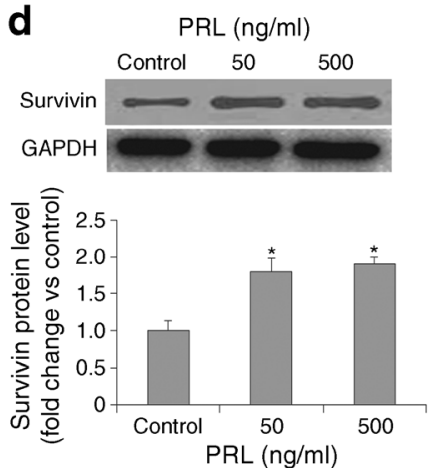
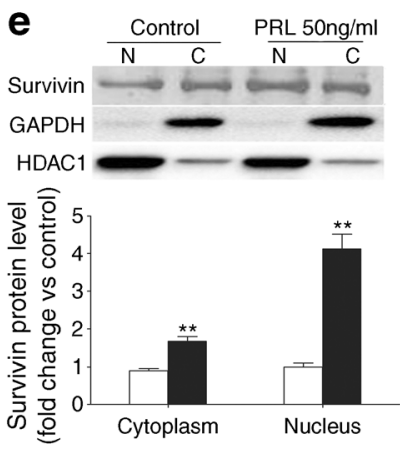
Impaired beta cell mass expansion with glucose intolerance in pregnant survivin-KO mice To determine whether survivin is necessary for beta cell mass expansion during pregnancy, we used the RIP-Cre-Survivin ${ }^{\mathrm{fl} / \mathrm{fl}}$ (KO) mice, with C57BL/6 and littermate RIP-Cre-Survivin ${ }^{+/+}$(WT) mice as controls. The body weight and pancreas weight were similar between WT and KO mice from NP state to P18.5 (ESM Fig. 1). We next examined glucose homeostasis in these mice. Although KO and WT mice had similar glucose tolerance in the NP state, they displayed slightly higher glucose excursion than C57BL/6 mice (Fig. 3a). However, at P14.5, KO mice had developed significantly impaired glucose tolerance while WT and C57BL/6 mice displayed similar glucose excursion (Fig. 3b). Circulating insulin levels following a glucose bolus were significantly lower in $\mathrm{KO}$ pregnant mice at P14.5 than in WT and C57BL/6 mice (Fig. 3c) but ITTs showed no change in peripheral insulin sensitivity in the three groups (Fig. 3d), suggesting that impaired glucose tolerance at P14.5 was due to inadequate supply of insulin. GSIS from isolated islets was markedly higher at P14.5 than in the NP state, but there was no significant difference between the three groups, indicating that the inadequate supply of insulin in $\mathrm{KO}$ mice was attributed to the decline in beta cell mass (Fig. 3e).

Analysis of the pancreatic islet morphometry showed that beta cell mass gradually increased from the NP state to P18.5 in WT and C57BL/6 mice, due to an increase in both cell number and cell size (Fig. $4 \mathrm{a}-\mathrm{c}$ ). However, the expansion of beta cell mass was diminished in $\mathrm{KO}$ mice, with a significant reduction in total beta cell number (Fig. 4a, b). Interestingly, the beta cell size was largest in KO mice in both the NP state and at P18.5, but no increment in cell size was observed when comparing P18.5 with the NP state (Fig. 4c). The increment in total islet number was significantly attenuated in the mutant mice; this was attributed mainly to the lack of increase in the number of larger islets in the KO mice while the increment in the number of smaller islets was similar to that seen in WT mice (Fig. 4d). From the NP state to P14.5, the percentage of Ki67-positive beta cells was increased by almost threefold in WT and C57BL/6 mice but failed to increase in the KO mice (Fig. 4e). The percentage of TUNEL-positive beta cells was similar among the three groups of mice at P14.5 (Fig. 4f). Taken together, the absence of survivin impairs proliferation of existing beta cells leading to impaired beta cell mass expansion during pregnancy. PRL directly stimulates beta cell proliferation and this effect is diminished without survivin.

To gain insight into potential cell cycle abnormalities during pregnancy, quantitative real- time PCR analysis for genes involved in cell cycle progression in maternal islets was performed. In WT mice, there was a marked upregulation of Cdk4/Ccnd1, Cdk2/Ccne1, Cdk1/Ccnb1, Rb1, E2f1, p53 (also known as Trp53) and $p 21$ (also known as $C d k n 1 a$ ) expression with significant downregulation of p16 (also known as $C d k n 2 a$ ) at P14.5, compared with the NP state, coinciding with cell cycle progression during pregnancy (Fig. 4g). However, KO mice displayed a significant attenuation in the increase in Cdk4/Ccnd1, E2f1, p53 and p21 expression at P14.5 when compared with WT controls, while the change
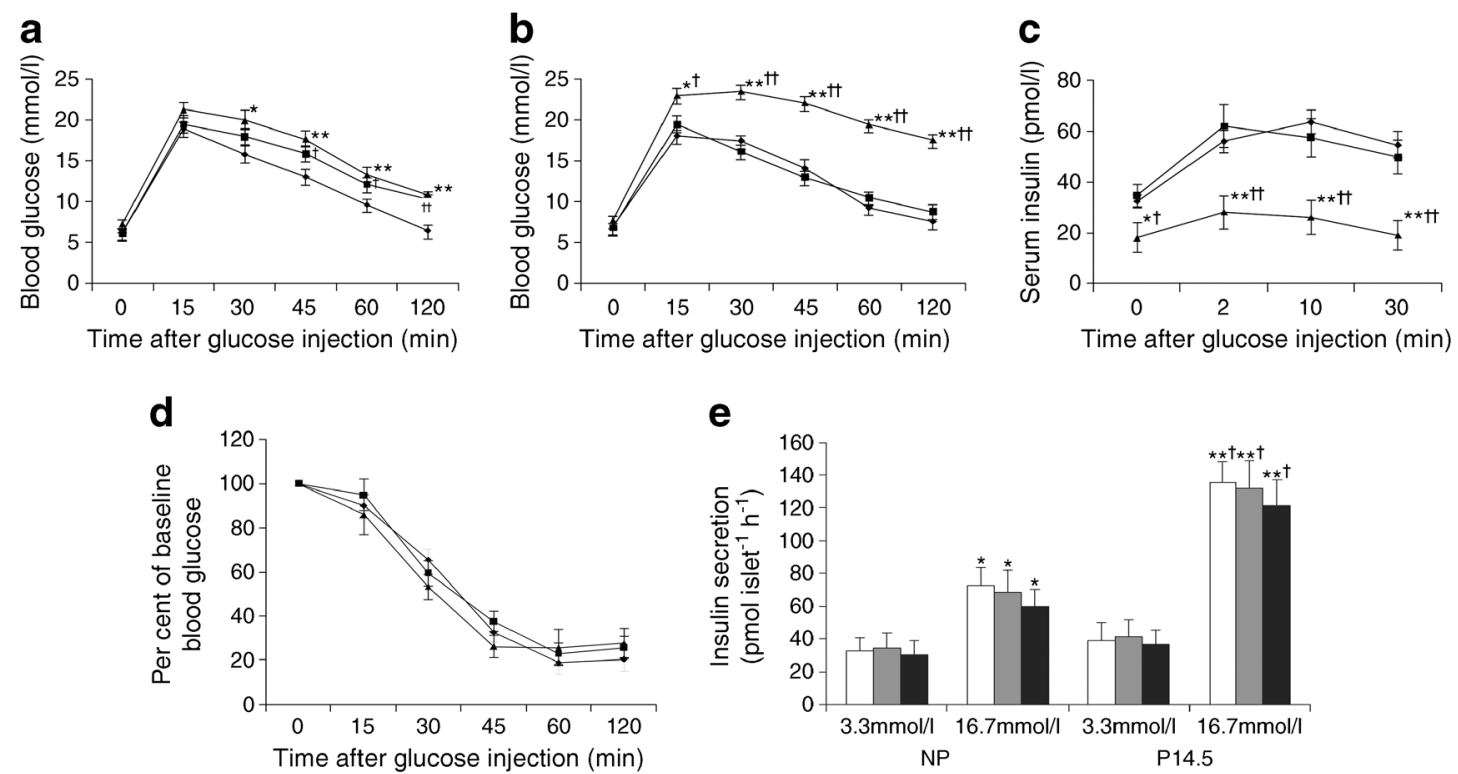

e

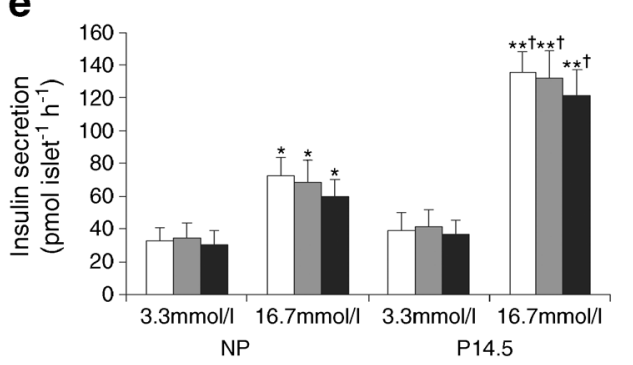

${ }^{*} p<0.05$ and $* * p<0.01$ vs WT mice; ${ }^{\dagger} p<0.05$ and ${ }^{\dagger \dagger} p<0.01$ vs C57BL/6 mice. (e) GSIS of islets isolated from KO (black bars), WT (grey bars) and C57BL/6 mice (white bars) in the NP state and at P14.5. ${ }^{*} p<0.05$ and $* * p<0.01$ vs $3.3 \mathrm{mmol} / \mathrm{l} ;{ }^{\dagger} p<0.05$ vs NP $16.7 \mathrm{mmol} / \mathrm{l}$. The data are presented as means $\pm \operatorname{SEM}(n=8)$ 
a

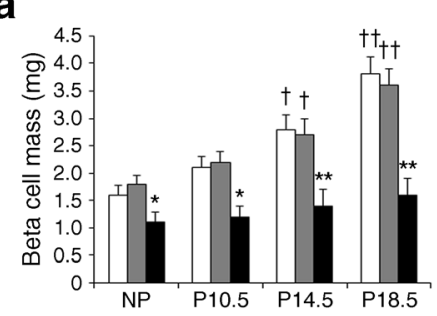

d

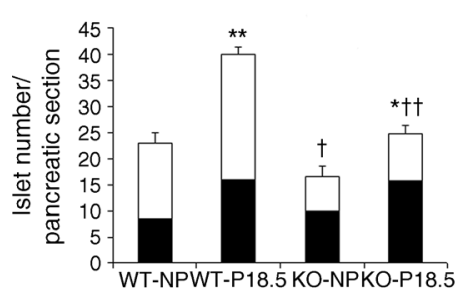

g b

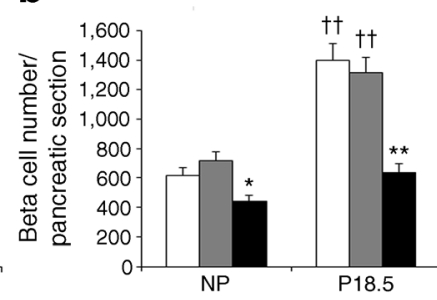

e

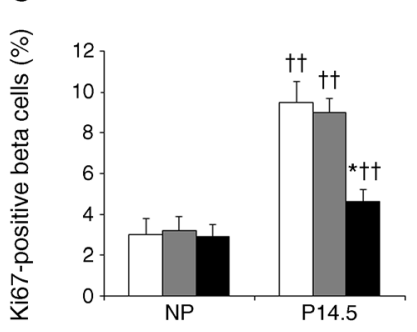

C

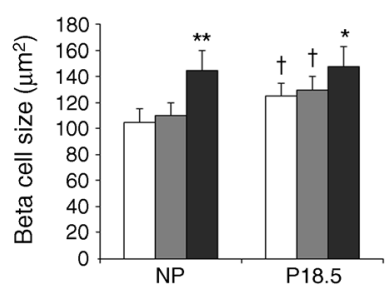

f

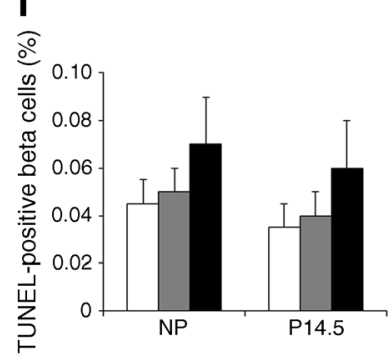

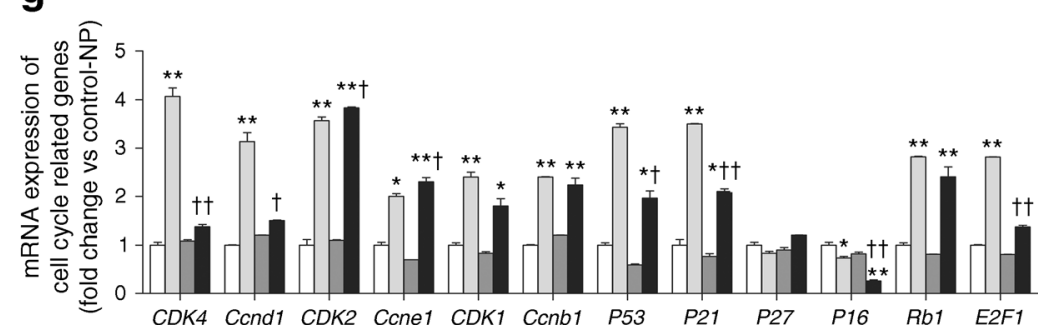

Fig. 4 Beta cell mass, proliferation and apoptosis in RIP-Cre-Survivin ${ }^{\mathrm{fl} / \mathrm{fl}}$ mice during pregnancy. (a) Beta cell mass of RIP-Cre-Survivin ${ }^{\mathrm{fl} / \mathrm{fl}}(\mathrm{KO}$, black bars), RIP-Cre-Survivin ${ }^{+++}$(WT, grey bars) and C57BL/6 mice (white bars) in the NP state and at P10.5, P14.5 and P18.5. (b, c) Beta cell number (b) and cell size (c) of KO (black bars), WT (grey bars) and C57BL/6 (white bars) mice at P18.5. (d) Islet number of KO and WT mice at P18.5. White bars, larger islets ( $>10$ nuclei); black bars, small islets (1-10 nuclei) (e, f) Percentage of Ki67-positive (e) and TUNEL- positive (f) beta cells of KO (black bars), WT (grey bars) and C57BL/6 (white bars) mice at P14.5. (g) Quantitative real-time PCR analyses of cell cycle-related genes from islets of KO and WT mice in the NP state and at P14.5. White bars, WT mice in the NP state; light-grey bars, WT mice at P14.5; dark-grey bars, KO mice in the NP state; black bars, $\mathrm{KO}$ mice at P14.5. The data are presented as means $\pm \operatorname{SEM}(n=5) .{ }^{*} p<0.05$ and ${ }^{* *} p<0.01$ vs NP; ${ }^{\dagger} p<0.05$ and ${ }^{\dagger \dagger} p<0.01$ vs other counterparts

diminished in the survivin siRNA group, which showed a persistently high percentage of cells in $\mathrm{G} 0 / \mathrm{G} 1$, no change in $\mathrm{S}$ phase and significantly low levels of cells in G2/M phase when compared with the control siRNA or blank control groups (Fig. 5d-f). These data suggest that knockdown of survivin led INS-1 cells to arrest in G1/S phase. Analysis of cell cycle genes in control groups showed markedly increased Cdk4/Ccnd1, Cdk1/Ccnb1, Cdk2/Ccne1, Rb1, E2f1, p53 and $p 21$ expression but significantly decreased $p 16$ expression after PRL stimulation (Fig. $5 \mathrm{~g}$ ), indicating that these genes might have contributed to PRL-stimulated INS-1 cell proliferation. In the survivin siRNA group, compared with corresponding controls, the increments in $C d k 4 / C c n d 1, E 2 f 1, p 53$ and $p 21$ levels were attenuated after PRL stimulation, with significant elevation of $C d k 2 / C c n e 1$ and downregulation of p16 mRNA (Fig. $5 \mathrm{~g}$ ), further confirming the vital role of survivin in PRL-mediated beta cell proliferation.

PRL-stimulated survivin expression through multiple signalling pathways During pregnancy, multiple signalling 
a
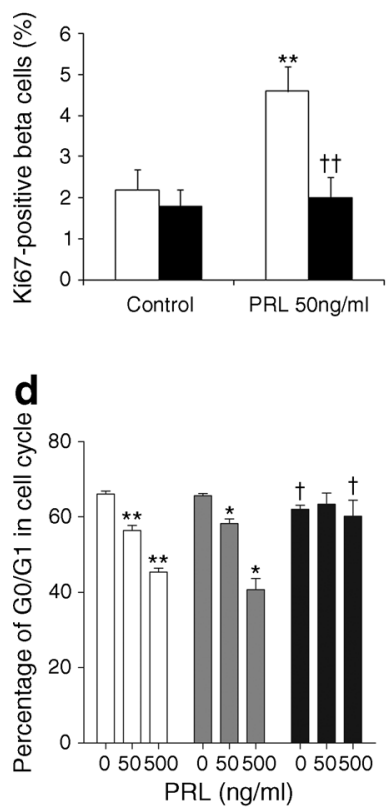

b
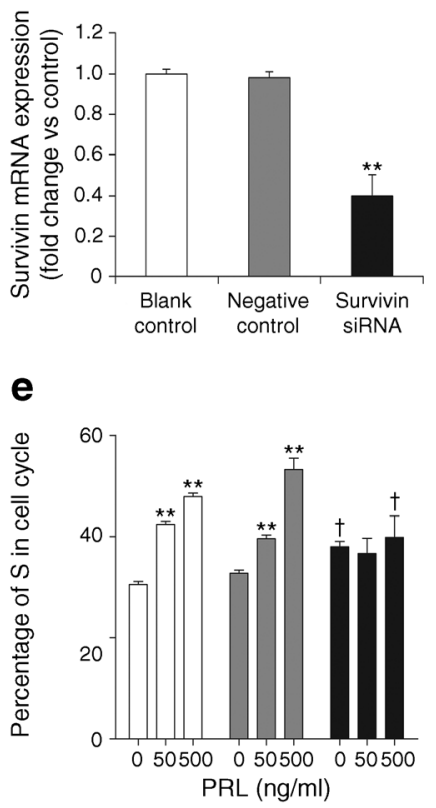
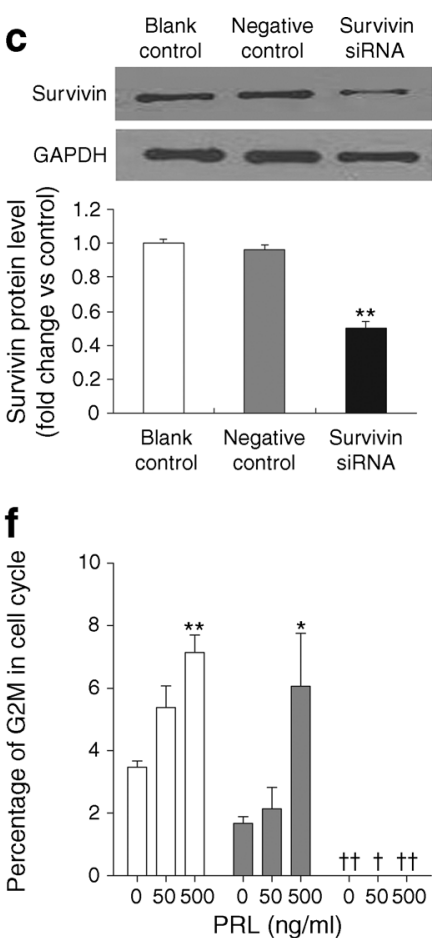

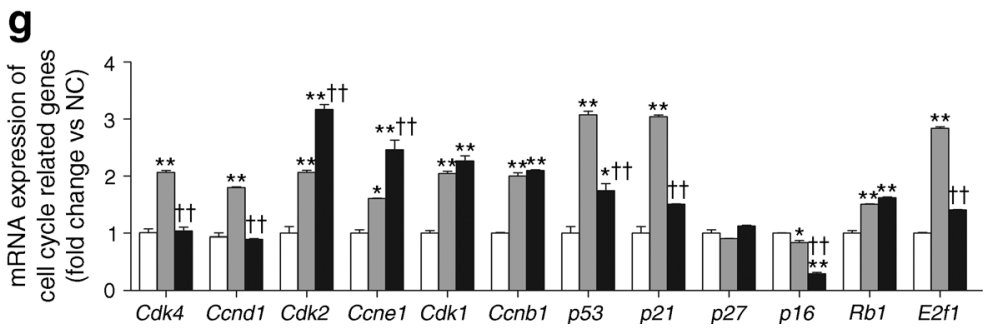

Fig. 5 Survivin absence blocks PRL-mediated beta cell proliferation. (a) Percentage of Ki67-positive beta cells in islets isolated from RIP$\mathrm{Cre}_{\text {-Survivin }}{ }^{\mathrm{fl} / \mathrm{fl}}(\mathrm{KO})$ and $\mathrm{RIP}$-Cre-Survivin ${ }^{+/+}$(WT) mice following stimulation with PRL for $24 \mathrm{~h}$. White bars, WT mice; black bars, KO mice. ${ }^{* *} p<0.01$ vs WT control; ${ }^{\dagger \dagger} p<0.01$ vs WT + PRL. (b) Level of survivin mRNA after transfection with siRNA against survivin. INS-1 cells were untransfected (blank control, white bars) or were transfected with non-silencing control siRNA (negative control, grey bars) or with siRNA against survivin (survivin siRNA, black bars). ${ }^{* *} p<0.01$ vs negative control. (c) Western blots and quantification showing decreased levels of survivin in INS-1 cells after siRNA transfection. GAPDH was

pathways such as JAK2-STAT5, ERK1/2, PI3K and FOXM1 are activated in rodent maternal islets $[7,28]$. We found that the protein levels of p-Akt, p-STAT5, p-ERK1/2 and FOXM1 were markedly increased in mouse maternal islets at P14.5 as well as in PRL-stimulated INS-1 cells (Fig. 6a, b). To further examine the role of survivin in PRL-stimulated signalling pathways, inhibition studies in PRL-stimulated INS-1 cells were performed. Specific inhibitors of Akt, STAT5, PIM and ERK pathways significantly decreased the protein levels of FOXM1 and survivin in PRL-stimulated INS-1 cells (Fig. 6c-f), revealing that FOXM1 and survivin are the common downstream effectors of these pathways in response to PRL (Fig. 6g). used as control. ${ }^{* *} p<0.01$ vs negative control. (d-f) Aberrant cell cycle progression in INS-1 cells after survivin knockdown and PRL stimulation for $24 \mathrm{~h}$. White bars, blank control; grey bars, negative control; black bars, survivin siRNA. ${ }^{*} p<0.05$ and $* * p<0.01$ vs no PRL; ${ }^{\dagger} p<0.05$ and ${ }^{\dagger \dagger} p<0.01$ vs negative control. (g) Quantitative real-time PCR analyses of cell cycle-related genes in INS-1 cells after survivin knockdown and PRL stimulation for $24 \mathrm{~h}$. White bars, negative control; grey bars, negative control+PRL; black bars, survivin siRNA+PRL. ${ }^{*} p<0.05$ and $* * p<0.01$ vs negative control; ${ }^{\dagger} p<0.05$ and ${ }^{\dagger \dagger} p<0.01$ vs negative + PRL control. The data are presented as means $\pm \operatorname{SEM}(n=3-5)$

\section{Discussion}

Survivin has been shown not only to be transiently expressed within pancreatic islets during embryogenesis and the neonatal period but also to be induced in adult islets during chronic pancreatitis or type $1 \mathrm{~A}$ diabetes in humans, or after partial PDL in mice [14-16, 29-31], suggesting the vital role of survivin in beta cell mass regulation. In this study, we revealed the pattern of survivin expression in mouse maternal islets during pregnancy and defined the essential role of survivin in PRL-mediated pancreatic beta cell proliferation.

During pregnancy, the expression of survivin gradually increased from P10.5, peaked at P14.5 and then declined by 

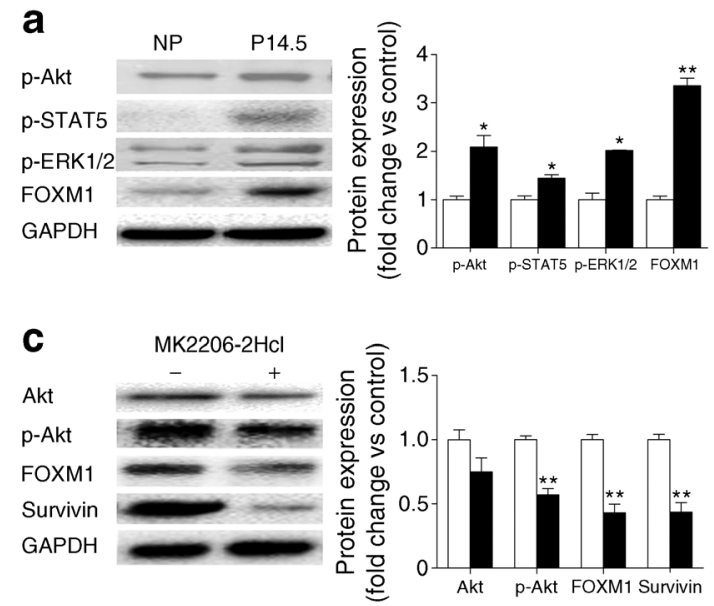

e
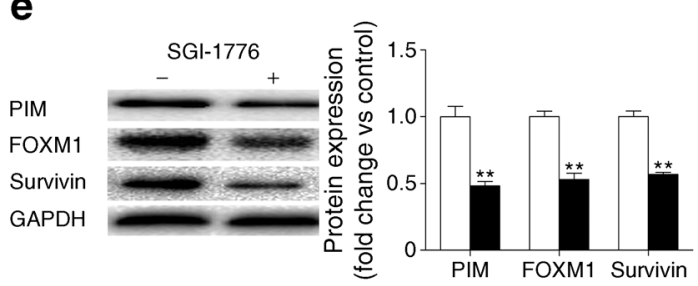

\section{b}
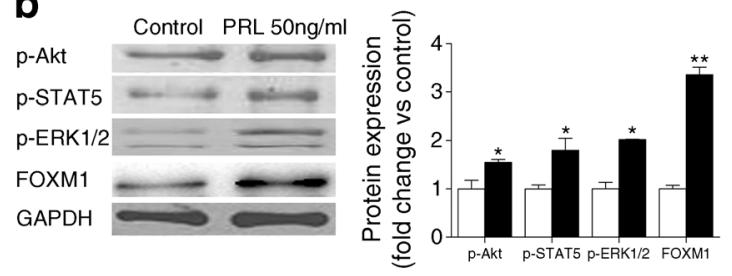

d
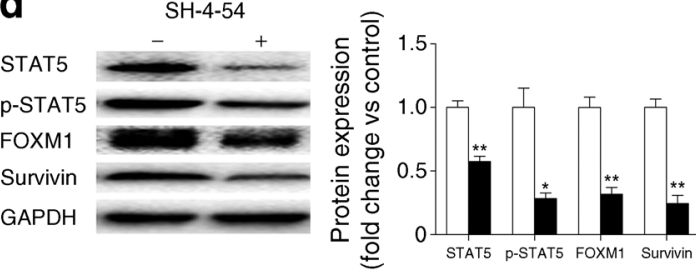

f
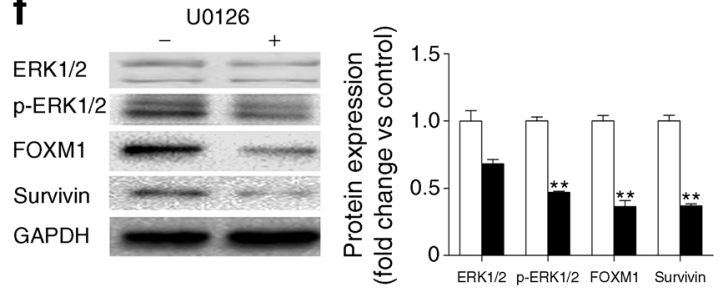

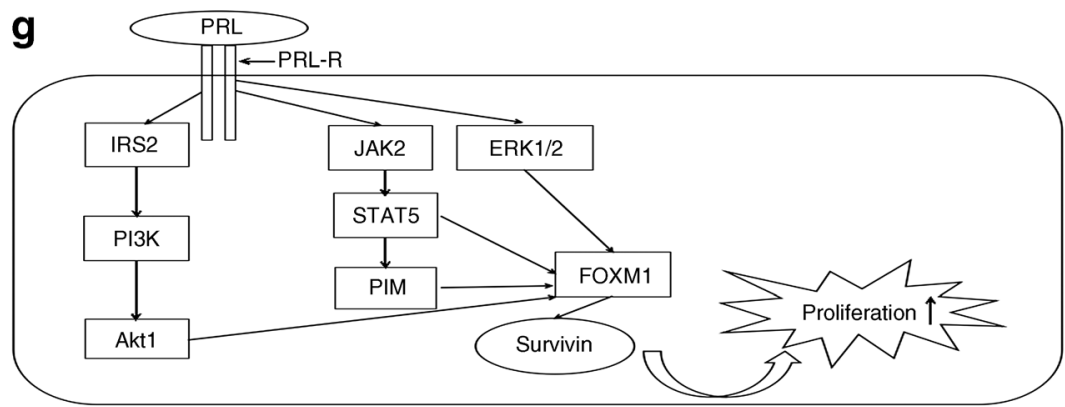

Fig. 6 Signalling pathways of PRL-stimulated beta cell proliferation. (a) Expression of p-Akt, p-STAT5, p-ERK1/2 and FOXM1 in maternal islets from mice in the NP state (white bars) and during pregnancy at P14.5 (black bars). ${ }^{*} p<0.05$ and ${ }^{* *} p<0.01$ vs NP. (b) Expression of p-Akt, pSTAT5, p-ERK1/2 and FOXM1 in INS-1 cells, unstimulated (control, white bars) or stimulated with PRL (50 ng/ml, black bars) for $24 \mathrm{~h}$. (cf) Expression of indicated proteins in PRL-stimulated INS-1 cells without

P18.5; this pattern closely mirrors gestational islet cell proliferation trends [23]. The pregnant RIP-Cre-Survivin ${ }^{\mathrm{f} / \mathrm{fl}}$ mice developed glucose intolerance, compromised beta cell mass expansion and impaired beta cell proliferation, with an attenuated increase in $C d k 4 / C c n d 1, E 2 f 1, p 53$ and $p 21$ expression at P14.5. In the early G1 phase cyclin-dependent kinase (CDK) 4/cyclin (CCN) D1 can directly phosphorylate Rb, which releases E2F1 and leads to transcriptional activity for cell cycle progression [32]. $p 21$ is the target gene of $\mathrm{p} 53$, and p21 protein serves not only as a cell cycle inhibitor [33, 34] but also as a promoter of cell cycle progression $[35,36]$, promoting mitosis by forming a complex with caspase- 3 and survivin [37]. Therefore, attenuation of the increased expression of $C d k 4 / C c n d 1, E 2 f 1, p 53$ and $p 21$ in mutant islets may (control, white bars) or with (black bars) Akt inhibitor (MK2206-2HCl, c), STAT inhibitor (SH-4-54, d), PIM inhibitor (SGI-1776, e) or ERK1/2 inhibitor (U0126, f). The data are presented as means \pm SEM $(n=5)$. $*_{p}<0.05$ and $* * p<0.01$ vs control. (g) Diagram illustrating the signalling pathways of FOXM1/survivin in the regulation of PRL-stimulated beta cell proliferation

lead to cell cycle delay. Meanwhile, the changes in Cdk2/Ccnel and $p 16$ expression were more pronounced in the KO mice. During G1/S phase, Cdk2/Ccnel and Cdk4/ Ccndl can serve partially overlapping functions [38] and the effects of CDK2/CCNE1 can occur independently of the $\mathrm{Rb} /$ E2F1 pathway [39]. Therefore, upregulation of $C d k 2 / C c n e 1$ and downregulation of the cell cycle inhibitor p16 during pregnancy could be an adaptive change in an attempt to overcome the defects in cell cycle progression in mutant islets. In addition, beta cell apoptosis in the NP state was similar to that at P14.5 in these mice, which is consistent with our previous reports $[14,16]$, indicating that apoptosis did not significantly contribute to the defect in gestational beta cell mass expansion in the mutant mice. 
Previous studies have demonstrated convincingly that activation of the PRL-R is critical for the occurrence of pregnancy-associated changes in beta cell mass and function $[1,27]$. Single-nucleotide polymorphisms of the PRLR gene 5' UTR and promoter region are associated with increased risk for gestational diabetes mellitus in humans [40]. We found that PRL could significantly promote beta cell proliferation in islets and INS-1 cells. Exposure of INS-1 cells to PRL markedly increased nuclear localisation of survivin with cell cycle progression to $\mathrm{S}$ and $\mathrm{G} 2 / \mathrm{M}$ phase. Survivin transcription increases during $\mathrm{G} 1$ and reaches a peak in $\mathrm{G} 2 / \mathrm{M}$ [15]. Targeted deletion of survivin in INS-1 cells resulted in cell cycle disturbance with an arrest in G1/S phase after PRL stimulation. The compromised induction of cell cycle genes, such as $C d k 4 / C c n d 1, E 2 f 1, p 53$ and $p 21$, was similar to changes in islets of maternal $\mathrm{KO}$ mice, further confirming the vital role of survivin in PRL-mediated beta cell proliferation.

To define the signalling pathway upstream of survivin that might regulate beta cell mass expansion during pregnancy, we analysed several potential pathways. Consistent with the findings of other rodent studies [7, 28], levels of p-Akt, p-STAT5, p-ERK1/2 and FOXM1 were markedly increased in maternal islets at P14.5 as well as in PRL-stimulated INS-1 cells. FOXM1, an essential factor expressed during $\mathrm{S}$ phase, was involved in regulation of proliferation by binding with $\mathrm{G} 2 / \mathrm{M}$ regulators [41]. Survivin has been found to be an essential downstream target of FOXM1 [42]. Inhibitors of the Akt, STAT5, PIM or ERK pathway significantly decreased the expression of survivin and FOXM1 in PRL-stimulated INS-1 cells. This reveals that FOXM1/survivin is the common downstream effector of Akt, STAT5, PIM and ERK pathways during PRL-stimulated beta cell proliferation. In addition, PRL-R activation could lead to transactivation of the EGF receptor, which activates MAPK and PI3K-Akt-mTOR pathways to stimulate survivin gene expression [18].

Some limitations to our study include the presence of pre-existing decrease in beta cell mass of female $\mathrm{KO}$ mice that could potentially impact on the observed effects during gestation. The use of an inducible Cre model such as the $M I P-C r e-E R$ mouse may have circumvented this limitation. Also, since RIP-Cre is expressed to some degree in brain tissue [43], the extent to which survivin in brain contributes to glucose homeostasis during pregnancy remains to be determined. Finally, Brouwers et al recently revealed that the RIP-Cre mouse model produces human growth hormone (hGH), which binds to the PRL-R leading to pregnancy-like changes in the islets [44]. However, our data in mice showed beta cell mass, insulin secretion and beta cell proliferation to be comparable between the RIPCre mice and C57BL/6 mice during pregnancy. This discrepancy might be due to the different level of expression of transgenic hGH in the islet in various background strains in addition to different potential environmental factors.
Taken together, our data reveal that transient induction of survivin in pancreatic beta cells during pregnancy is essential for beta cell mass expansion. Survivin directly participates in beta cell proliferation during pregnancy and the FOXM1survivin pathway functions downstream of Akt, STAT5PIM and ERK in PRL-stimulated beta cell proliferation. These results clarify the molecular effectors of the signalling pathways downstream of PRL-R activation that have therapeutic implications for the expansion of beta cell mass in the setting of diabetes treatment.

Acknowledgements We thank H. Okada (Division of Signaling Biology, Ontario Cancer Institute, Toronto, ON, Canada) for providing the Survivin $^{\mathrm{fl} / \mathrm{fl}}$ mice. We thank X. Han (Key Laboratory of Human Functional Genomics of Jiangsu Province, Department of Biochemistry and Molecular Biology, Nanjing Medical University, Nanjing, China) for providing the INS-1 cell line.

Funding This work is supported by grants to $\mathrm{X}$. Wu from the National Natural Science Foundation of China (81070621 and 81261120566), the Scientific Research Foundation for the Returned Overseas Chinese Scholars, State Human Resource Ministry and State Education Ministry, International Science and Technology Cooperation Plan - the Key National and Regional Research and Development Cooperation Projects (BZ2011042), Jiangsu Province key medical personnel project (RC2011068). MW is supported by grants from the Canadian Institutes of Health Research Operating Grants (MOP-81148 and CCI-125690) and Canada Research Chair in Signal Transduction in Diabetes Pathogenesis.

Duality of interest The authors declare that there is no duality of interest associated with this manuscript.

Contribution statement All authors contributed to the study conception and data collection and analysis, and approved the final version of the manuscript. YX, X. Wang and LG contributed to the generation and analyses of research data and to preparation of the manuscript. JZ, HZ and HS contributed to the generation of research data and reviewed the manuscript. MW and X. Wu designed experiments, contributed to the discussion and interpretation of the data and reviewed and edited the manuscript. X. Wu is the guarantor of this work.

\section{References}

1. Sorenson RL, Brelje TC (1997) Adaptation of islets of Langerhans to pregnancy: beta-cell growth, enhanced insulin secretion and the role of lactogenic hormones. Horm Metab Res 29:301-307

2. Parsons JA, Brelje TC, Sorenson RL (1992) Adaptation of islets of Langerhans to pregnancy: increased islet cell proliferation and insulin secretion correlates with the onset of placental lactogen secretion. Endocrinology 130:1459-1466

3. Van Assche FA, Aerts L, De Prins F (1978) A morphological study of the endocrine pancreas in human pregnancy. Br J Obstet Gynaecol 85:818-820

4. Butler AE, Cao-Minh L, Galasso R et al (2010) Adaptive changes in pancreatic beta cell fractional area and beta cell turnover in human pregnancy. Diabetologia 53:2167-2176

5. Kim C, Newton KM, Knopp RH (2002) Gestational diabetes and the incidence of type 2 diabetes: a systematic review. Diabetes Care 25:1862-1868 
6. Brelje TC, Stout LE, Bhagroo NV, Sorenson RL (2004) Distinctive roles for prolactin and growth hormone in the activation of signal transducer and activator of transcription 5 in pancreatic islets of Langerhans. Endocrinology 145:4162-4175

7. Amaral ME, Cunha DA, Anhe GF et al (2004) Participation of prolactin receptors and phosphatidylinositol 3-kinase and MAP kinase pathways in the increase in pancreatic islet mass and sensitivity to glucose during pregnancy. J Endocrinol 183:469-476

8. Amaral ME, Ueno M, Carvalheira JB et al (2003) Prolactin-signal transduction in neonatal rat pancreatic islets and interaction with the insulin-signaling pathway. Horm Metab Res 35:282-289

9. Hugl SR, Merger M (2007) Prolactin stimulates proliferation of the glucose-dependent beta-cell line INS-1 via different IRS-proteins. JOP 8:739-752

10. Friedrichsen BN, Galsgaard ED, Nielsen JH, Moldrup A (2001) Growth hormone- and prolactin-induced proliferation of insulinoma cells, INS-1, depends on activation of STAT5 (signal transducer and activator of transcription 5). Mol Endocrinol 15: 136-148

11. Mita AC, Mita MM, Nawrocki ST, Giles FJ (2008) Survivin: key regulator of mitosis and apoptosis and novel target for cancer therapeutics. Clin Cancer Res 14:5000-5005

12. Altieri DC (2008) Survivin, cancer networks and pathway-directed drug discovery. Nat Rev Cancer 8:61-70

13. Altieri DC (2008) New wirings in the survivin networks. Oncogene 27:6276-6284

14. Wu X, Wang L, Schroer S et al (2009) Perinatal survivin is essential for the establishment of pancreatic beta cell mass in mice. Diabetologia 52:2130-2141

15. Jiang Y, Nishimura W, Devor-Henneman D et al (2008) Postnatal expansion of the pancreatic beta-cell mass is dependent on survivin. Diabetes 57:2718-2727

16. Wu X, Zhang Q, Wang X et al (2012) Survivin is required for betacell mass expansion in the pancreatic duct-ligated mouse model. Plos One 7:e41976

17. Rieck S, White P, Schug J et al (2009) The transcriptional response of the islet to pregnancy in mice. Mol Endocrinol 23:1702-1712

18. Hakonen E, Ustinov J, Palgi J, Miettinen PJ, Otonkoski T (2014) EGFR signaling promotes beta-cell proliferation and survivin expression during pregnancy. Plos One 9:e93651

19. Nguyen KT, Tajmir P, Lin CH et al (2006) Essential role of Pten in body size determination and pancreatic beta-cell homeostasis in vivo. Mol Cell Biol 26:4511-4518

20. Liadis N, Murakami K, Eweida M et al (2005) Caspase-3dependent beta-cell apoptosis in the initiation of autoimmune diabetes mellitus. Mol Cell Biol 25:3620-3629

21. Xue Y, Liu C, Xu Y et al (2010) Study on pancreatic islet adaptation and gene expression during pregnancy in rats. Endocrine 37:83-97

22. Wang H, Gambosova K, Cooper ZA et al (2010) EGF regulates survivin stability through the Raf-1/ERK pathway in insulinsecreting pancreatic beta-cells. BMC Mol Biol 11:66

23. Rieck S, Kaestner KH (2010) Expansion of beta-cell mass in response to pregnancy. Trends Endocrinol Metab 21:151-158

24. Weinhaus AJ, Stout LE, Bhagroo NV, Brelje TC, Sorenson RL (2007) Regulation of glucokinase in pancreatic islets by prolactin: a mechanism for increasing glucose-stimulated insulin secretion during pregnancy. J Endocrinol 193:367-381

25. Parsons JA, Bartke A, Sorenson RL (1995) Number and size of islets of Langerhans in pregnant, human growth hormoneexpressing transgenic, and pituitary dwarf mice: effect of lactogenic hormones. Endocrinology 136:2013-2021
26. Freemark M, Avril I, Fleenor D et al (2002) Targeted deletion of the PRL receptor: effects on islet development, insulin production, and glucose tolerance. Endocrinology 143:1378-1385

27. Huang C, Snider F, Cross JC (2009) Prolactin receptor is required for normal glucose homeostasis and modulation of beta-cell mass during pregnancy. Endocrinology 150:1618-1626

28. Zhang H, Zhang J, Pope CF et al (2010) Gestational diabetes mellitus resulting from impaired beta-cell compensation in the absence of FoxM1, a novel downstream effector of placental lactogen. Diabetes 59:143-152

29. Gianani R, Campbell-Thompson M, Sarkar SA et al (2010) Dimorphic histopathology of long-standing childhood-onset diabetes. Diabetologia 53:690-698

30. Hasel C, Bhanot UK, Heydrich R, Strater J, Moller P (2005) Parenchymal regression in chronic pancreatitis spares islets reprogrammed for the expression of NFKB and IAPs. Lab Invest $85: 1263-1275$

31. Liggins C, Orlicky DJ, Bloomquist LA, Gianani R (2003) Developmentally regulated expression of survivin in human pancreatic islets. Pediatr Dev Pathol 6:392-397

32. Chen HZ, Tsai SY, Leone G (2009) Emerging roles of E2Fs in cancer: an exit from cell cycle control. Nat Rev Cancer 9:785-797

33. Chen J, Jackson PK, Kirschner MW, Dutta A (1995) Separate domains of $\mathrm{p} 21$ involved in the inhibition of Cdk kinase and PCNA. Nature 374:386-388

34. Goubin F, Ducommun B (1995) Identification of binding domains on the p21Cip1 cyclin-dependent kinase inhibitor. Oncogene 10: 2281-2287

35. LaBaer J, Garrett MD, Stevenson LF et al (1997) New functional activities for the p21 family of CDK inhibitors. Genes Dev $11: 847-862$

36. Cheng M, Olivier P, Diehl JA et al (1999) The p21(Cip1) and p27(Kip1) CDK 'inhibitors' are essential activators of cyclin Ddependent kinases in murine fibroblasts. EMBO J 18:1571-1583

37. Li F, Ackermann EJ, Bennett CF et al (1999) Pleiotropic celldivision defects and apoptosis induced by interference with survivin function. Nat Cell Biol 1:461-466

38. Ezhevsky SA, Nagahara H, Vocero-Akbani AM, Gius DR, Wei MC, Dowdy SF (1997) Hypo-phosphorylation of the retinoblastoma protein $(\mathrm{pRb})$ by cyclin $\mathrm{D}: \mathrm{Cdk} 4 / 6$ complexes results in active pRb. Proc Natl Acad Sci U S A 94:10699-10704

39. Resnitzky D, Reed SI (1995) Different roles for cyclins D1 and E in regulation of the G1-to-S transition. Mol Cell Biol 15:3463-3469

40. Le TN, Elsea SH, Romero R, Chaiworapongsa T, Francis GL (2013) Prolactin receptor gene polymorphisms are associated with gestational diabetes. Genet Test Mol Biomarkers 17:567-571

41. Kalin TV, Ustiyan V, Kalinichenko VV (2011) Multiple faces of FoxM1 transcription factor: lessons from transgenic mouse models. Cell Cycle 10:396-405

42. Wang IC, Chen YJ, Hughes D et al (2005) Forkhead box M1 regulates the transcriptional network of genes essential for mitotic progression and genes encoding the SCF (Skp2-Cks1) ubiquitin ligase. Mol Cell Biol 25:10875-10894

43. Gannon M, Shiota C, Postic C, Wright CV, Magnuson M (2000) Analysis of the Cre-mediated recombination driven by rat insulin promoter in embryonic and adult mouse pancreas. Genesis 26:139-142

44. Brouwers B, de Faudeur G, Osipovich AB et al (2014) Impaired islet function in commonly used transgenic mouse lines due to human growth hormone minigene expression. Cell Metab 20: 979-990 